\title{
Preparation of Chitosan-coated Magnetite Nanoparticles by Sonochemical Method for MRI Contrast Agent
}

\author{
Jun Hee Cho ${ }^{1}$, Sang Gil Ko ${ }^{2}$, Yangkyu $\mathrm{Ahn}^{3 *}$, and Eun Jung Choi ${ }^{4}$ \\ ${ }^{1}$ Quantum Optics Division, Korea Atomic Energy Research Institute, Deajeon 305-353, Korea \\ ${ }^{2}$ Environment \& Resources Research Center, Korea Research Institute of Chemical Technology, Deajeon 305-353, Korea \\ ${ }^{3}$ Department of Chemistry, Konyang University, Chungnam 320-711, Korea \\ ${ }^{4}$ Department of Opthalmic Optics, Konyang University, Daejeon 320-718, Korea
}

(Received 15 January 2009, Received in final form 31 July 2009, Accepted 4 August 2009)

\begin{abstract}
Magnetic nanoparticles were synthesized by using the sonochemical method with oleic acid as a surfactant. The average size of the magnetite nanoparticles was controlled by varying the ratio $\mathrm{R}=\left[\mathrm{H}_{2} \mathrm{O}\right] /[$ surfactant $]$ in the range of 2 to $9 \mathrm{~nm}$. To prepare chitosan-coated magnetite nanoparticles, chitosan solution was added to a magnetite colloid suspension under ultrasonication at room temperature for $20 \mathrm{~min}$. The chitosan-coated magnetite nanoparticles were characterized by several techniques. Atomic force microscopy (AFM) was used to image the chitosan-coated nanoparticles. Magnetic hysteresis measurement was performed by using a superconducting quantum interference device (SQUID) magnetometer to investigate the magnetic properties of the magnetite nanoparticles and the chitosan-coated magnetite nanoparticles. The SQUID measurements revealed the superparamagnetism of both nanoparticles. The T1- and T2-weighted MR images of these chitosan-coated magnetite colloidal suspensions were obtained with a $4.7 \mathrm{~T}$ magnetic resonance imaging (MRI) system. The chitosancoated magnetite colloidal suspensions exhibited enhanced MRI contrasts in vitro.
\end{abstract}

Keywords : magnetite, sonochemical method, chitosan, MRI contrast agent

\section{Introduction}

Magnetic nanoparticles have interesting magnetic and electrical properties. They are used in permanent magnets, magnetic memory devices, and a new magnetic refrigeration system [1]. Aqueous magnetic fluids composed of magnetite nanoparticles covered with biocompatible functionalized shells have been increasingly used for diagnostics and therapy in biomedical applications and biotechnology. The important properties of magnetic nanoparticles for biomedical applications are biocompatibility, injectability, high-level accumulation in the target tissue or organ, and, most importantly, non-toxicity [2, 3]. They can be used in the targeted delivery of therapeutic agents in vivo, in the hyperthermic treatment of cancers, in magnetic resonance imaging (MRI) as contrast agents and in the biomagnetic separations of biomolecules [4, 5].

MRI is an effective and widely used medical diagnostic technique. To date, gadolinium or manganese salts and superparamagnetic iron oxide-based particles are by far

*Corresponding author: Tel: +82-41-730-5584

Fax: +82-41-736-5762, e-mail: ykahn@konyang.ac.kr the most commonly used materials as MRI contrast agents. As gadolinium is highly toxic, contrast agents utilize a chelating molecule to hold the gadolinium ion tightly and securely, thereby ensuring rapid excretion via the kidney. Superparamagnetic iron oxide contrast agents have the advantage of producing an enhanced proton relaxation in MRI better than those produced by paramagnetic ions. Consequently, the human body requires a smaller dose of superparamagnetic iron oxide agent than paramagnetic agent $[6,7]$.

Chitosan is a natural polysaccharide and can be extracted by a deacetylation process from chitin, an abundant matter from crustacean shells. Due to the large number of hydroxyl and amino groups in its structure, chitosan can thus be modified by various chemical reactions. Due to its non-toxic, biocompatible and slower biodegradable characteristics, chitosan has been widely used as a drug delivery and biomedical material for artificial skin and wound healing bandage applications [8, 9].

In this work, magnetite nanoparticles obtained by the sonochemical method were successfully dispersed in water and coated with chitosan in an aqueous medium to afford stable and biocompatible colloidal suspensions that could 
be used as MRI contrast agents.

\section{Experiments}

Ferric chloride hexahydrate $\left(\mathrm{FeCl}_{3} \cdot 6 \mathrm{H}_{2} \mathrm{O}\right)$ and ferrous chloride tetrahydrate $\left(\mathrm{FeCl}_{2} \cdot 4 \mathrm{H}_{2} \mathrm{O}\right)$ were purchased from Merck, oleic acid and acetic acid $\left(\mathrm{CH}_{3} \mathrm{COOH}\right)$ from Samchun, and tetramethylammonium hydroxide (TMAOH) and chitosan from Acros. All chemicals used were reagent grade.

A mixed solution of $0.15 \mathrm{M} \mathrm{FeCl}_{2}(30 \mathrm{ml}, 7.5 \mathrm{mM})$ and $0.30 \mathrm{M} \mathrm{FeCl}_{3}(30 \mathrm{ml}, 15.0 \mathrm{mM})$ was prepared for synthesis of uniform magnetite nanoparticles. Then, oleic acid was added to the solution with the molar ratio $\mathrm{R}=\left[\mathrm{H}_{2} \mathrm{O}\right]$ / [surfactant] in the range of 95 to $133.1 .2 \mathrm{M} \mathrm{TMAOH} \mathrm{(60}$ $\mathrm{ml}, 72 \mathrm{mM}$ ) solution was rapidly added with ultrasonic waves on an ultrasonic probe (Ulsso Hitech Co. Ltd., Model ULH700S, $10 \mathrm{~mm}$, Ti horn, $20 \mathrm{kHz}$ ). The black precipitations $\left(\mathrm{Fe}_{3} \mathrm{O}_{4}\right.$ : magnetite) obtained were washed 3 times using ultracentrifugation. The average particle size and size distribution of the precipitate nanoparticles were measured with the dynamic laser scattering, (DLS; PSS) method. The morphologies and mean size were examined with atomic force microscopy (AFM; PSIA, XE-100). The wet precipitates were dried in a vacuum oven at $80^{\circ} \mathrm{C}$ to characterize the crystal structure and magnetic properties using X-ray diffraction (XRD; Philips, X"pert MPD) and a superconducting quantum interference device (SQUID; Quantum design-MPMS5).

To prepare the magnetite colloid, nanoparticles were dispersed in weak alkali solution $(\mathrm{pH} 9)$, by ultrasonic irradiation for $10 \mathrm{~min}$. Chitosan $0.07 \%(\mathrm{w} / \mathrm{v})$ solution was prepared with $0.17 \mathrm{M}$ acetic acid solution. To prepare the chitosan-coated magnetite, the chitosan acetic acid solution was added dropwise to magnetite colloidal suspensions of equal volume under ultrasonication at room temperature for $20 \mathrm{~min}$. The FT-IR spectra of the magnetite and chitosan-coated magnetite were measured on a SHIMADZU FTIR-8400S spectrophotometer by the potassium bromide pellet method. The iron (Fe) concentration of this stock solution was $0.20 \mathrm{M}$ and the stock solution was diluted to (ED this $0.2 \mathrm{M}$ concentration is not a dilution, this is the stock solution) $0.02 \mathrm{M}, 0.002 \mathrm{M}, 0.2 \mathrm{mM}$ and $0.02 \mathrm{mM}$. The T1- and T2-weighted MRI images of these chitosancoated magnetite colloidal suspensions were obtained with a 4.7 T MRI system (Bruker, BioSpec 47/40).

\section{Results and Discussion}

The XRD pattern of the sample shown in Fig. 1 revealed its crystalline spinel ferrite crystal structure. No extra

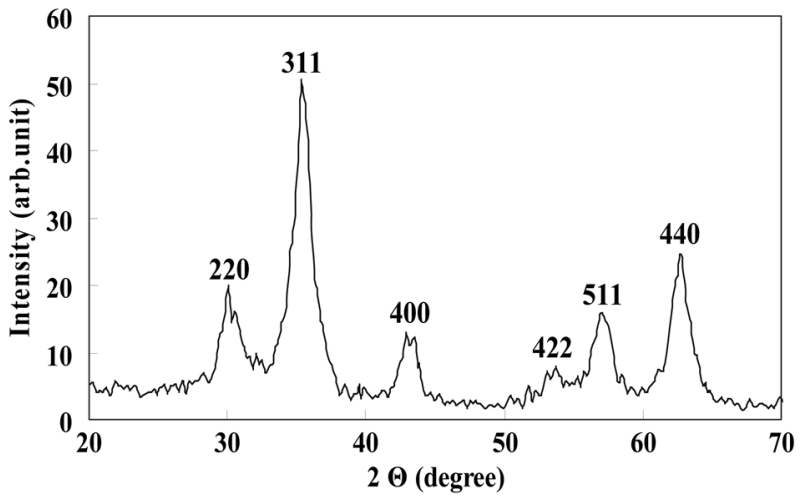

Fig. 1. XRD results of the nanoparticle powder synthesized by sonochemical method.

Table 1. Average sizes of the magnetite nanoparticles prepared from the sonochemical method with oleic acid.

\begin{tabular}{ccccc}
\hline \hline \multirow{2}{*}{ Magnetite } & $\begin{array}{c}\mathrm{R}=[\text { water]/ } \\
\text { [surfactant] }\end{array}$ & $\begin{array}{c}\text { Average } \\
\text { size (nm) }\end{array}$ & $\begin{array}{c}\text { Distribution } \\
\text { (\%) }\end{array}$ & $\begin{array}{c}\text { S. Dev } \\
\text { (nm/\%) }\end{array}$ \\
\cline { 2 - 5 } nano & (a) 95 & 2.2 & 100.0 & $0.3 / 13.3$ \\
Particles & (b) 104 & 4.1 & 99.5 & $0.4 / 11.2$ \\
& (c) 114 & 5.6 & 97.9 & $0.5 / 9.8$ \\
& (d) 133 & 9.0 & 94.1 & $0.8 / 10.8$ \\
\hline
\end{tabular}

crystalline structure was detected. Each peak in the pattern could be indexed on a spinel crystal structure.

The average size of the magnetite nanoparticles was controlled by varying the ratio $\mathrm{R}=\left[\mathrm{H}_{2} \mathrm{O}\right] /[$ surfactant $]$ in the range of 2 to $9 \mathrm{~nm}$, as shown in Table 1 . The average particle size and size distribution of the magnetite nanoparticles were measured with the DLS method. The samples prepared by sonochemical method with oleic acid showed a narrow size distribution. With increasing [water]/[oleic acid] molar ratio, the average size of the magnetite nanoparticles increased. Considering their uniform size and narrow distributions, these magnetite nanoparticles were suitable for the preparation of ferrofluids for biomedical

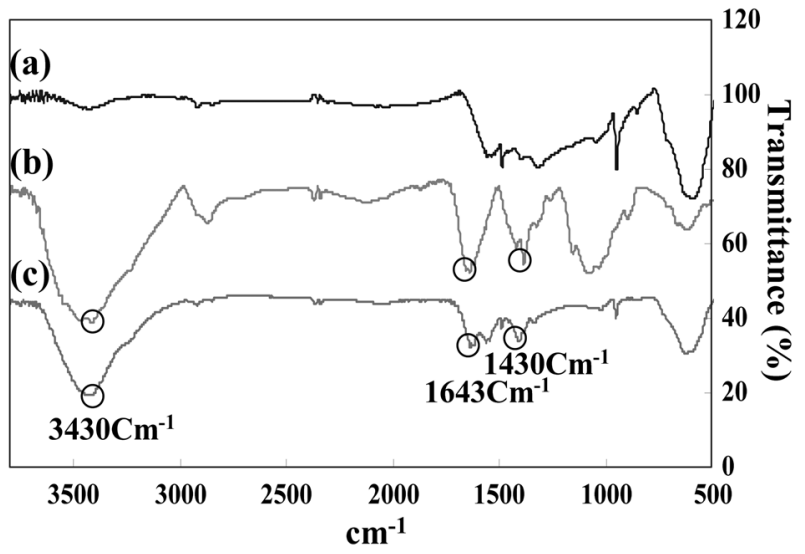

Fig. 2. FT-IR spectra of (a) magnetite, (b) chitosan and (c) chitosan-coated magnetite. 


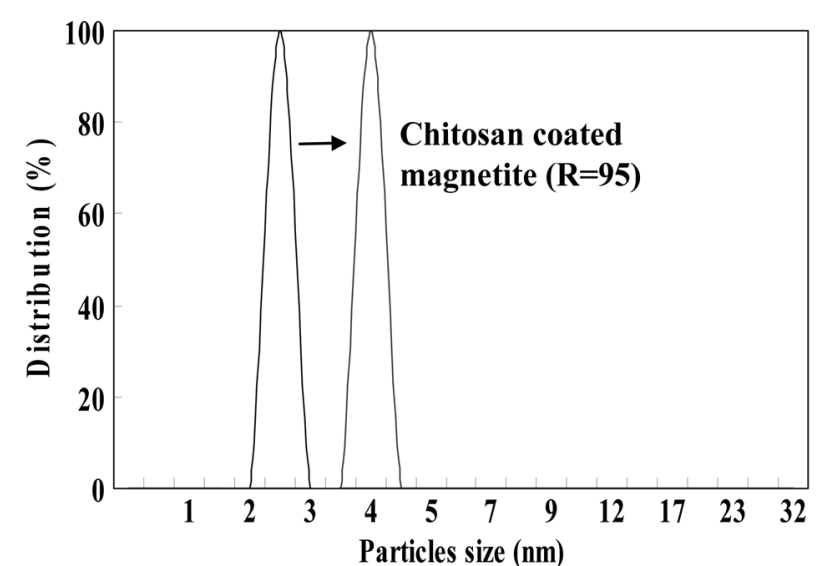

Fig. 3. Change of size distribution of the chitosan-coated magnetite nanoparticles.

applications.

FT-IR analysis confirmed the presence of chitosan-coating layers on the surface of the magnetite nanoparticles, as shown in Fig. 2, together with the spectra of the magnetite and chitosan used as a reference. Characteristic absorption bands of chitosan were observed in the chitosancoated magnetite. The FT-IR spectra of the chitosancoated magnetite exhibited the characteristic absorption bands of chitosan $\left(\mathrm{O}-\mathrm{H}\right.$ stretch, $\mathrm{N}-\mathrm{H}$ bend and $\mathrm{C}-\mathrm{H}_{2}$ bend: $3430 \mathrm{~cm}^{-1}, 1643 \mathrm{~cm}^{-1}$ and $1430 \mathrm{~cm}^{-1}$, respectively). The chitosan-coated magnetite had bands of chitosan as well as magnetite. These results revealed the coating of the magnetite by chitosan.

Fig. 3 and Table 2 show the particle distributions of the magnetite nanoparticles and the chitosan-coated magnetite nanoparticles. The chitosan layer was barely observed by transmission electron microscopy (TEM) because of its low contrast, but it could be estimated by the DLS method.
Table 2. Size distributions of the chitosan-coated magnetite nanoparticles.

\begin{tabular}{cccc}
\hline \hline \multirow{2}{*}{$\begin{array}{c}\text { [water]/ } \\
\text { [surfactant] }\end{array}$} & $\begin{array}{c}\text { Magnetite } \\
\text { nanoparticles } \\
\text { size (nm) }\end{array}$ & \multicolumn{2}{c}{ chitosan-coated magnetite } \\
\cline { 3 - 4 } & 2.2 & $\begin{array}{c}\text { Average } \\
\text { size (nm) }\end{array}$ & S. Dev (nm/\%) \\
\hline $\mathrm{R}=95$ & 4.1 & 4.1 & $0.4 / 11.5$ \\
$\mathrm{R}=104$ & 5.8 & 5.6 & $0.6 / 12.1$ \\
$\mathrm{R}=114$ & 9.0 & 7.7 & $0.8 / 10.3$ \\
$\mathrm{R}=133$ & & 10.5 & $1.1 / 10.8$ \\
\hline
\end{tabular}

The average size of the magnetite and the chitosan-coated magnetite was $2.2 \mathrm{~nm}$ at $\mathrm{R}=95$ and $4.1 \mathrm{~nm}$, respectively, as shown in Fig. 3. The chitosan-coated magnetite nanoparticles were $1.9 \mathrm{~nm}$ larger than the magnetite nanoparticles.

The magnetite nanoparticles and the chitosan-coated magnetite nanoparticles synthesized at $\mathrm{R}=133$ were spherical with a mean diameter of about $6.4 \mathrm{~nm}$ and $10.7 \mathrm{~nm}$, as shown in the AFM images of Fig. 4(a) and (b), respectively. The AFM images show that the magnetite nanoparticles were coated by chitosan.

Superparamagnetic relaxation is an essential requirement for the use of magnetite nanoparticles as an MRI contrast enhancement agent. The magnetization curves for both nanoparticles are shown in Fig. 5. Fig 5(a) shows a typical magnetization curve of the magnetite nanoparticles with a magnetization value of $46 \mathrm{emu} / \mathrm{g}$. Both remanence and coercivity were zero, suggesting that the magnetite nanoparticles were superparamagnetic. Fig. 5(b) shows a magnetization curve of the chitosan-coated magnetite nanoparticles. The absence of hysteresis in the magnetization indicated that the chitosan-coated magnetite nanoparticles were also superparamagnetic with a magnetization value of $45 \mathrm{emu} / \mathrm{g}$. Both nanoparticles showed superparamag-
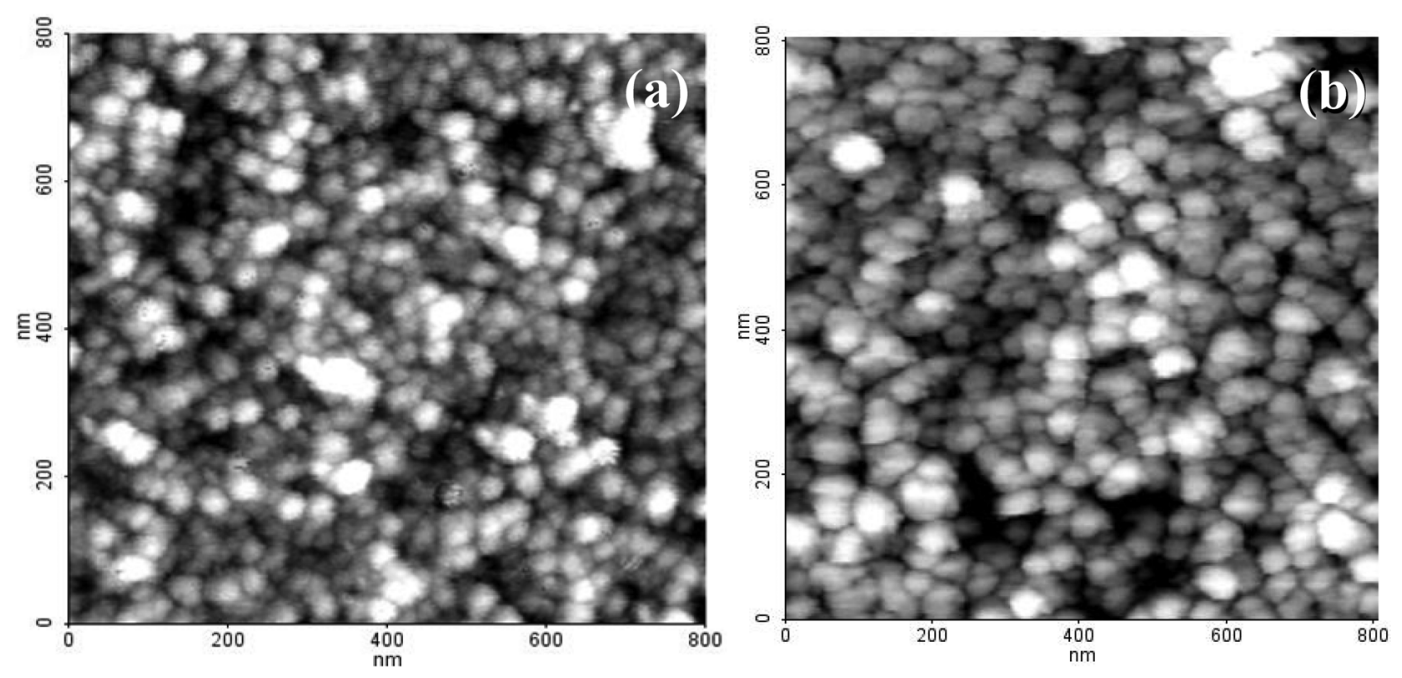

Fig. 4. AFM images of the magnetite nanoparticles (a) and the chitosan-coated nanoparticles (b). 


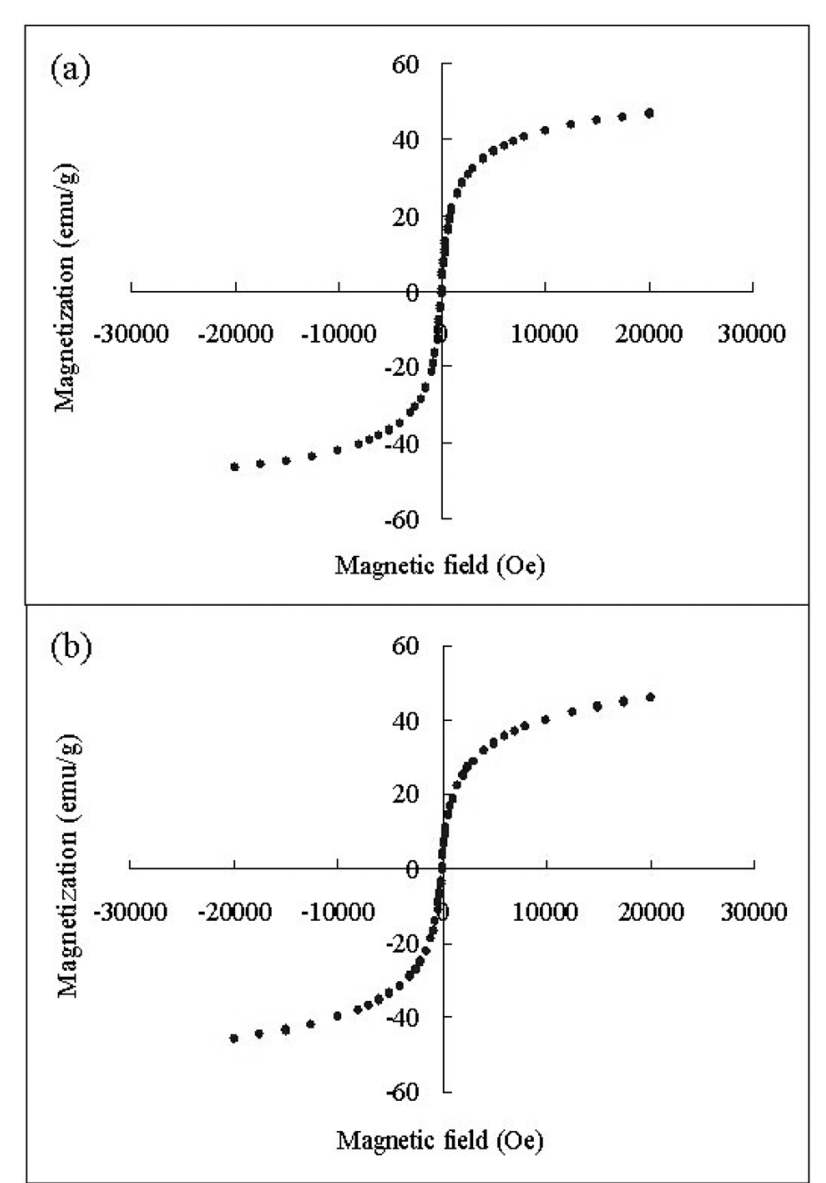

Fig. 5. Magnetic field dependence of the magnetization at room temperature: (a) the magnetite nanoparticles, and (b) the chitosan-coated magnetite nanoparticles.

netism at room temperature. The uniform size, narrow distributions and superparamagnetism of these chitosancoated magnetite nanoparticles demonstrated their suitability for MRI contrast agent applications.

The T1- and T2-weighted MR images of the chitosancoated magnetite colloidal suspensions with a Fe-concentration ranging from $0.2 \mathrm{M}$ to $0.02 \mathrm{mM}$ are shown in Fig. 6. These suspensions were stable for 15 days without precipitation. At the $\mathrm{Fe}$ concentration of $0.2 \mathrm{mM}$, the MRI contrast was markedly enhanced compared with that of the other concentrations. The chitosan-coated magnetite colloidal suspensions demonstrated potential as an MRI contrast agent.

\section{Conclusion}

We synthesized uniform magnetite nanoparticles using chemical coprecipitation technique through the sonochemical method with oleic acid as the surfactant. The average size of the magnetite nanoparticles could be controlled by varying the molar ratio $\mathrm{R}=\left[\mathrm{H}_{2} \mathrm{O}\right] /[$ surfactant $]$ in

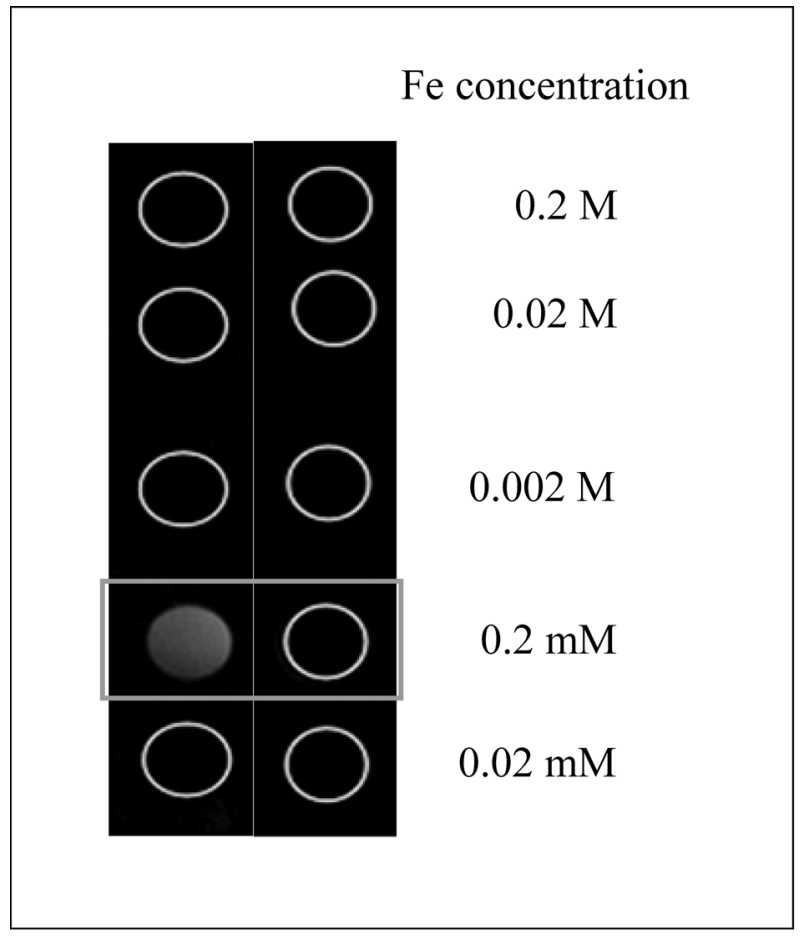

Fig. 6. T1- (left) and T2-weighted image (right) of the chitosan-coated magnetite nanoparticle colloidal suspensions.

the range of 2.2 to $9.0 \mathrm{~nm}$. These magnetite nanoparticles were well coated by chitosan to make ferrofluids. Both the magnetite nanoparticles and the chitosan-coated nanoparticles exhibited superparamagnetism at room temperature. Of the chitosan-coated magnetite colloidal suspensions, the $0.2 \mathrm{mM} \mathrm{Fe}$ solution showed the best MRI contrast and was stable without precipitation. Therefore, the chitosan-coated magnetite colloidal suspension has a promising potential as an MRI contrast agent.

\section{Acknowledgment}

This work was supported by the Korea Energy Management Coperation.

\section{References}

[1] X. M. Lin and C. S. Samia, J. Magn. Magn. Mater. 305, 100 (2006).

[2] A. Ito, M. Shinkai, H. Honda, and T. Kobayashi, J. Biosci. Bioeng. 100(6), 1 (2005).

[3] Y. Deng, L. Wang, W. Yang, S. Fu, and A Ela.ýssari, J. Magn. Magn. Mater. 257, 69 (2003).

[4] Yong Jae Suh, Dae Sup Kil, Kang Sup Chung, Hyo Sook Lee, and Huiping Shao, J. Magnetics 13, 106 (2008).

[5] A. K. Gupta and M. Gupta, Biomater. 26, 3995 (2005).

[6] E. H. Kim, H. S. Lee, B. K. Kwak, and B. K. Kim, J. 
Magn. Magn. Mater. 289, 328 (2005).

[7] M.C. Bautista, O. B. Miguel, X. Zhao, M. P. Morales, T G. Carreno, R. P. Alejo, J. R. Cabello, and S. V. Verdaguer, Institute of Physics Publishing; Nanotechnology, vol 15, pp. S154-S159 (2004).
[8] S. Baxter, S. Zivanovic, and J. Weiss, Food Hydrocolloids 19, 821 (2005).

[9] Y. C. Chang and D. H. Chen, J. Colloid and Interface Science 283, 446 (2005). 